\title{
A Compact Wideband CMOS Low-Noise Amplifier Using Shunt Resistive-Feedback and Series Inductive-Peaking Techniques
}

\author{
Hsien-Ku Chen, Da-Chiang Chang, Ying-Zong Juang, and Shey-Shi Lu, Senior Member, IEEE
}

\begin{abstract}
A wideband low-noise amplifier (LNA) with shunt resistive-feedback and series inductive-peaking is proposed for wideband input matching, broadband power gain and flat noise figure (NF) response. The proposed wideband LNA is implemented in 0.18- $\mu \mathrm{m}$ CMOS technology. Measured results show that power gain is greater than $10 \mathrm{~dB}$ and input return loss is below $-10 \mathrm{~dB}$ from 2 to $11.5 \mathrm{GHz}$. The IIP3 is about $+3 \mathrm{dBm}$, and the NF ranges from 3.1 to $4.1 \mathrm{~dB}$ over the band of interest. An excellent agreement between the simulated and measured results is found and attributed to less number of passive components needed in this circuit compared with previous designs. Besides, the ratio of figure-ofmerit to chip size is as high as $190\left(\mathrm{~mW}^{-1} / \mathrm{mm}^{2}\right)$ which is the best results among all previous reported CMOS-based wideband LNA.
\end{abstract}

Index Terms-CMOS, feedback, low cost, low-noise amplifier (LNA), series peaking, small chip size, wideband.

\section{INTRODUCTION}

W IDEBAND systems have recently gained much attention due to their capability of high data rate transmission. The so called ultrawideband (UWB) technology provides attractive high speed wireless signaling schemes, which use the frequency bands in $3.1 \sim 10.6 \mathrm{GHz}$. To interface with the antenna and pre-select filter, the low-noise amplifier (LNA) input impedance should be close to $50 \Omega$ across the band from 3.1 to $10.6 \mathrm{GHz}$.

Up to now, many wideband techniques have been proposed for the UWB LNA. The distributed amplifier (DA) is widely used for wideband application due to its intrinsic broadband frequency response going all the way down to dc along with good input and output impedance matching. Yet, so far, high power consumption and large die area have hampered its widespread applications [1], [2]. Recently, a cascode CMOS LNA with a bandpass response at the input for wideband impedance matching has been reported in [3]. The bandpass-filter (BPF) based topology incorporates the input impedance of the cascode amplifier as a part of the filter. However, the adoption of the filter at the input requires a number of reactive elements, which inevitably results in a larger chip area and noise figure

Manuscript received January 15, 2007; revised April 23, 2007. This work was supported by the National Science Council of Taiwan, R.O.C., under Grant NSC-96-2218E002-042.

H.-K. Chen and S.-S. Lu are with the Graduate Institute of Electronics Engineering, National Taiwan University, Taipei 100, Taiwan R.O.C. (e-mail: sslu@ntu.edu.tw).

D.-C. Chang and Y.-Z. Juang are with the Chip Implementation Center, National Applied Research Laboratories, Hsinchu 300, Taiwan, R.O.C.

Color versions of one or more of the figures in this letter are available online at http://ieeexplore.ieee.org.

Digital Object Identifier 10.1109/LMWC.2007.901797
(NF) degradation for on-chip implementation. An efficient way to achieve a broadband matching with small power dissipation and chip area is the common-gate input topology. However, it has been reported that a common-gate LNA exhibits a lower gain and higher NF than a common-source amplifier [4].

In this letter, a wideband LNA with wide bandwidth, low NF, low power consumption, broadband impedance matching and small chip-area is reported. Both the shunt resistive-feedback and series inductive-peaking techniques are adopted to design the $2 \sim 11.5 \mathrm{GHz}$ UWB LNA. These techniques have the merits of achieving wideband frequency response, sufficient power gain and small NF without using many passive components that require large die area.

\section{WIDEBAND LNA DESIGN}

The conventional cascode-type LNA with inductive sourcedegeneration is one of the most popular LNA topologies due to its merits of low power consumption, high gain, and high reverse isolation. For wideband application, this topology becomes unsuitable because the bandwidth of the input series resonance circuit is limited by its quality factor. Hence, a $3-5 \mathrm{GHz}$ cascode-type UWB LNA with resistive feedback [5] has been proposed to reduce the quality factor of the input series resonance circuit. But the bandwidth of that design is still limited by the quality factor of the input series resonance circuit and capacitive effect, namely, $C_{g s}, C_{g d}, C_{d b}$, and $C_{s b}$ of M1 and $\mathrm{M} 2$, and thus the input return loss and gain began to roll-off at 4.2 GHz. Fig. 1 shows the proposed UWB LNA, which contains the conventional cascode-type LNA enclosed in the dash lines of the figure. A smaller feedback resistor R2 $(300 \Omega)$ compared to that $(1 \mathrm{k} \Omega)$ of the conventional design [5] is used to reduce the quality factor of the input series resonance circuit further so that a wider input matching can be achieved. However, from the simulation, the feedback resistor R2 degrades the NF of LNA by approximately $1.2 \mathrm{~dB}$. Moreover, the power gain of this amplifier at higher frequencies is also degraded due to the capacitive effect of large size M1 and M2 chosen. To counterbalance this gain degradation, a series-peaking inductor L2 as shown in Fig. 1 is added to make the gain response flatter. The series-peaking inductor $\mathrm{L} 2$ can also resonate with the parasitic shunt capacitor $\mathrm{Cp}$ at the drain of M2. The value of $\mathrm{L} 2$ is chosen to be $1.13 \mathrm{nH}$ for peaking at the frequency of $10 \mathrm{GHz}$, and $\mathrm{C} 2$ is used for dc decoupling. The degeneration inductor TL1 was chosen to be small $(0.12 \mathrm{nH})$ for matching at higher band and implemented by a microstrip (MS) line with a line width of $6 \mu \mathrm{m}$ and a line-length of $162 \mu \mathrm{m}$. Compared to the spiral inductor, 


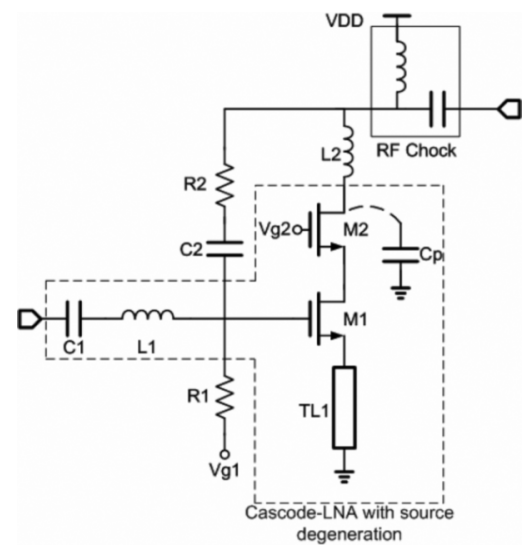

Fig. 1. Wideband LNA schematic.

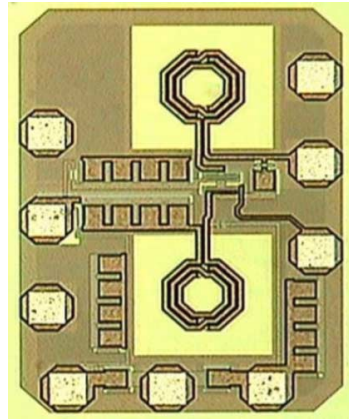

Fig. 2. Die photograph of the wideband LNA.

the MS inductor is less susceptible to process variation when the inductance is very small.

\section{EXPERIMENTAL RESULTS}

The wideband LNA was implemented in a 1P6M $0.18 \mu \mathrm{m}$ CMOS technology. The die photograph is shown in Fig. 2. The die area including testing pads is only $0.48 \times 0.69 \mathrm{~mm}^{2}$, which is very area-efficient and consequently cost effective due to the techniques adopted. The circuit was tested on wafer for RF characterization. The LNA draws $11.2 \mathrm{~mA}$ from bias-T and the drain voltages of M2 is $1.2 \mathrm{~V}$.

Fig. 3 shows the simulated and measured results of the input return loss and power gain of the wideband LNA. The power gain is above $10 \mathrm{~dB}$ with a $3-\mathrm{dB}$ frequency band up to $12.5 \mathrm{GHz}$ while the return losses are below $-10 \mathrm{~dB}$ from 2 to $11.5 \mathrm{GHz}$. Simulation shows that the bandwidth of power matching shrinks from $9.5 \mathrm{GHz}$ to only $\sim 2.5 \mathrm{GHz}$ if $\mathrm{R} 2$ is removed from the circuit. These results unequivocally prove the effectiveness of the broadband matching realized by using the small R2 shunt resistive-feedback technique in conjunction with the source degenerative inductior-TL1. Fig. 3 also shows the simulated frequency responses of the LNA with different values of L2. It is found that $\mathrm{L} 2=0.619 \mathrm{nH}$ corresponds to the maximally flat condition. In our work, $\mathrm{L} 2$ is chosen to be $1.13 \mathrm{nH}$ for peaking at $10 \mathrm{GHz}$. An L2 smaller than $1.13 \mathrm{nH}$ (but lager than $0.619 \mathrm{nH}$ ) can result in a flatter gain response (and even a wider bandwidth). However, in this work, a larger L2 value corresponding to under-damped rather than maximally flat condition is chosen

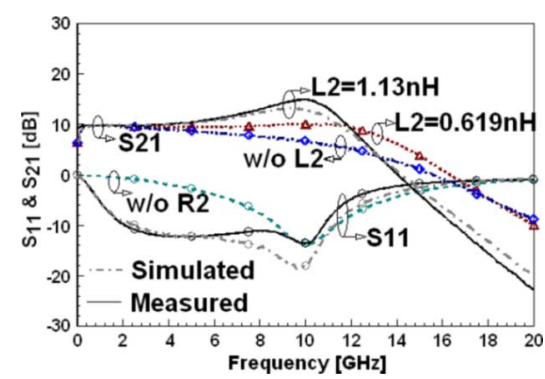

Fig. 3. Simulated and measured results for input return loss and power gain of wideband LNA versus frequency.

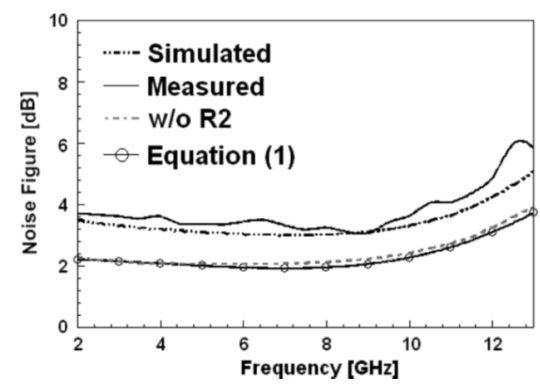

Fig. 4. Simulated and measured results for NF of wideband LNA versus frequency.

because the LNA is designed to be integrated with a mixer behind it and the intentional peaking at $10 \mathrm{GHz}$ can pre-compensate the usual overdamped response of the next stage. Besides, it can also be seen that the power gain begins to roll-off from $3 \mathrm{GHz}$ if L2 is removed from circuit, which evidences that the series inductive-peaking technique is useful for extending the bandwidth of the resistive feedback cascode-LNA.

Fig. 4 shows the simulated and measured results of the NF of this wideband LNA. Based on Fig. 4, the measured minimum and maximum NFs are $3.1 \mathrm{~dB}$ (at $9 \mathrm{GHz}$ ) and $4.1 \mathrm{~dB}$ (at $11.5 \mathrm{GHz}$ ), respectively, over the band of $2-11.5 \mathrm{GHz}$. From the simulation it is clear that the shunt feedback resistor R2 for the purpose of wideband matching degrades the NF of LNA by $\sim 1.2 \mathrm{~dB}$. Nevertheless, the achieved NFs are still better than the previously measured values of $3.1 \sim 10.6 \mathrm{GHz}$ CMOS LNAs [1]-[9]. In addition, this circuit also has the flattest NF across the band of all UWB LNAs, which is explained as follows. Based on [10] and neglecting gate noise, we can show that the NF of an LNA using a source degenerative inductor can be approximated by

$$
\mathrm{NF} \approx 1+\frac{R_{\text {par }}}{R_{S}}+\frac{\gamma}{\mathrm{G}_{M 1} R_{S}} \cdot\left|\left(\frac{s}{\omega_{\text {Onf }}}\right)^{2}+\frac{s}{\omega_{\mathrm{Onf}}} \frac{1}{Q_{\mathrm{IN}}}+1\right|^{2}
$$

where $R_{\mathrm{par}}$ and $Q_{\text {in }}=\omega_{O n f}(L 1+T L 1) /\left(R_{S}+\right.$ $\left.R_{\text {par }}\right)$ are the series parasitic resistance, and quality factor of the input $R-L-C$ circuit, respectively, $\omega_{O n f}=$ $1 / \sqrt{(L 1+T L 1)\left(C_{g s}+C_{g d}\right)}, R_{S}=50 \Omega$ and $\gamma$ the channel noise parameter. (1) is verified by comparison with simulation results (see Fig. 4). According to (1), the frequency responses (under-, over-, and critical-damping) of NF are controlled by $Q_{\text {in }}$ and in this work a $Q_{\text {in }}$ as close to the maximally flat condition as possible is chosen, which explains the excellent NF flatness of our LNA. 
TABLE I

RECENTLY REPORTED PERFORMANCES OF WIDEBAND LNAs

\begin{tabular}{|c|c|c|c|c|c|c|c|c|c|c|c|}
\hline & $\begin{array}{l}\text { Freq. } \\
{[\mathrm{GHz}]}\end{array}$ & $\begin{array}{c}\mathbf{S}_{11} \\
{[\mathrm{~dB}]}\end{array}$ & $\begin{array}{c}\text { Gain-max } \\
{[\mathrm{dB}]}\end{array}$ & $\begin{array}{c}\text { NF-min } \\
{[\mathrm{dB}]}\end{array}$ & $\begin{array}{c}\text { NF-flatness } \\
(\sim 3,1-10,6 \mathrm{GHz})\end{array}$ & $\begin{array}{c}\text { IIP3 } \\
{[\mathrm{dBm}]}\end{array}$ & $\left.\mid \begin{array}{c}\mathbf{P}_{1 \mathrm{~dB}} \\
{[\mathrm{dBm}]}\end{array}\right]$ & $\begin{array}{l}\text { Power } \\
{[\mathrm{mW}]}\end{array}$ & $\begin{array}{c}\text { Die Size } \\
{\left[\mathrm{mm}^{2}\right]}\end{array}$ & $\begin{array}{c}\text { FoM/Size } \\
{\left[(\mathrm{mW})^{-1} /\left(\mathrm{mm}^{2}\right)\right]}\end{array}$ & Technology \\
\hline [1], VLSI 2003 & $0.5 \sim 14$ & $<-11$ & 10.6 & 3.4 & $4.4 \pm 1$ & +20 & +10 & $52 @ 1.3-\mathrm{V}$ & 1.6 & $12.37 / 1.6=7.7$ & CMOS 0.18-um \\
\hline [2], JSSC & $0.04 \sim 7$ & $<-16$ & 8.6 & 5.7 & - & +3 & -8.5 & 9@1.3-V & 1.16 & $12.7 / 1.16=10.9$ & CMOS 0.18-um \\
\hline [3], JSSC & $2,3 \sim 9,2$ & $<-9.9$ & 10.4 & 4 & $6.75 \pm 2.75$ & -6.7 & -15 & 9@1.8-V & 1.1 & $28 / 1.1=25.5$ & CMOS 0.18-um \\
\hline${ }^{*}[4]$, CAS-I & $3.1 \sim 10.6$ & $<-9$ & 17.5 & 3.1 & $4.4 \pm 1.3$ & - & - & $33.2 @ 1.8-\mathrm{v}$ & 0.5 & $26.8 / 0.5=53.7$ & CMOS 0.18-um \\
\hline [5], JSSC & $2-4.6$ & $<-9$ & 9.8 & 2.3 & - & -7 & - & $12.6 @ 1.8-\mathrm{V}$ & 0.9 & $15.3 / 0.9=17$ & CMOS 0.18-um \\
\hline [6], ISSCC 2006 & $3 \sim 5$ & $<-10.5$ & 16 & 1.8 & - & -9 & -23 & 7.6@1.2-V & 0.63 & $54 / 0.63=85.6$ & CMOS 0.18-um \\
\hline [7], CICC 2005 & $1.2 \sim 11.9$ & $<-12$ & 9.7 & 4.5 & $6 \pm 1.5$ & -6.2 & - & $20 @ 1.8-\mathrm{V}$ & 0.59 & $14.9 / 0.59=25.3$ & CMOS 0.18-um \\
\hline [8], MWCL & $2 \sim 4.6$ & $<-10$ & 9.5 & 3.5 . & - & -0.8 & - & 16.5@1.5-V & 1.1 & $5.43 / 1.1=4.9$ & CMOS 0.13-um \\
\hline [9], RFIC 2006 & $3.1 \sim 10.6$ & $<-11.4$ & 12.02 & 4.7 & $5.15 \pm 0.45$ & -10 & -20 & $10.5 @ 1.5-\mathrm{V}$ & 0.665 & $24.7 / 0.665=37.1$ & CMOS 0.18-um \\
\hline This work & $2 \sim 11.5$ & $<-10$ & 14.8 & 3.1 & $3.58 \pm 0.41$ & +3 & -8.5 & 13.4@1.2-V & 0.33 & $62.8 / 0.33=190$ & CMOS 0.18-um \\
\hline
\end{tabular}

* [4] is the simulated results

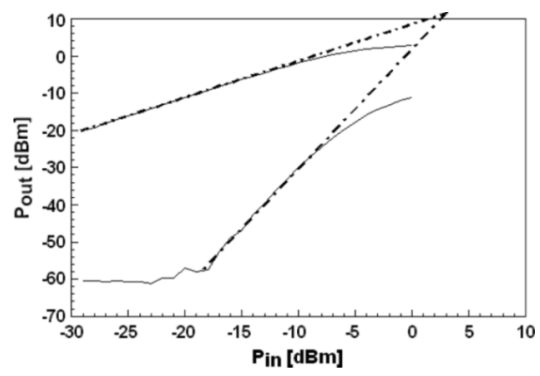

Fig. 5. Measured results for IIP3 under the two tones input of 5.2 and $5.8 \mathrm{GHz}$.

From Figs. 3 and 4, it is found that the simulation and measurement results agree very well, which is attributed to careful layout and less number of passive components needed in the proposed LNA compared with previous designs.

To characterize the nonlinear behavior, two-tone signals with equal power levels at 5.2 and $5.8 \mathrm{GHz}$, which are commonly used frequencies in wireless local area network applications, were applied to the proposed UWB LNA. The measured results in Fig. 5 indicate that this LNA has an $I I P_{3}$ of $3 \mathrm{dBm}$ and a $P_{1 \mathrm{~dB}}$ of $-8.5 \mathrm{dBm}$. These results demonstrate that the proposed wideband LNA achieves good linearity, too.

To evaluate the performance of UWB LNAs for their maximum power gain, $3 \mathrm{~dB}$-bandwidth, excess NF, power dissipation, and cut-off frequency (representing technology cost), the figure of merit (FoM) is defined as [11]

$$
\operatorname{FoM}\left[m W^{-1}\right]=\frac{S 21[1] \times B W[G H z]}{(N F-1)[1] \times P_{\mathrm{DC}}[m W] \times f_{t}[G H z]} .
$$

This FoM includes the most relevant parameters in order to evaluate a UWB LNA for low-cost and low-power applications. A summary of the state-of-the-art results is given in Table I. Our proposed LNA achieves the highest FoM of $62.8\left(\mathrm{~mW}^{-1}\right)$ among all CMOS-based wideband LNAs. Besides, the chip size of our LNA including testing pads is only $0.33 \mathrm{~mm}^{2}$, and thus the ratio of FoM to chip size can be even as high as $190\left(\mathrm{~mW}^{-1} / \mathrm{mm}^{2}\right)$, which is at least a factor of 2 better than any of the previously published results.

\section{CONCLUSION}

A compact wideband LNA for $2 \sim 11.5 \mathrm{GHz}$ application has been proposed. The shunt resistive-feedback in conjunction with series inductive-peaking provides a method for achieving a high performance wideband LNA with low cost. Because less component are used with this method, the proposed wideband LNA has shown a good agreement between simulation and measurement results. Besides, this wideband LNA reveals the best FoM of $62.8\left(\mathrm{~mW}^{-1}\right)$ and moreover the highest ratio $\left(190 \mathrm{~mW}^{-1} / \mathrm{mm}^{2}\right)$ of FoM to chip-size among all CMOS-based wideband LNAs.

\section{REFERENCES}

[1] R. Liu, C. Lin, K. Deng, and H. Wang, "A 0.5-14-GHz 10.6-dB CMOS cascode distributed amplifier," in VLSI Circuits Symp. Dig., Jun. 2003, vol. 17, pp. 139-140.

[2] F. Zhang and P. R. Kinget, "Low-power programmable gain CMOS distributed LNA," IEEE J. Solid-State Circuits, vol. 41, no. 6, pp. 1333-1343, Jun. 2006.

[3] A. Bevilacqua and A. M. Niknejad, "An ultrawideband CMOS lownoise amplifier for 3.1-10.6-GHz wireless receivers," IEEE J. SolidState Circuits, vol. 39, no. 12, pp. 2259-2268, Dec. 2004.

[4] Y. Lu, K. S. Yeo, A. Cabuk, and J. Ma, "A novel CMOS low-noise amplifier design for 3.1- to 10.6-GHz ultra-wide-band wireless receivers," IEEE Trans. Circuits Syst. I, vol. 53, no. 8, pp. 1683-1692, Aug. 2006.

[5] C. W. Kim, M. S. Kang, P. T. Anh, H. T. Kim, and S.-G. Lee, "An ultra-wideband CMOS low-noise amplifier for 3-5-GHz UWB system," IEEE J. Solid-State Circuits, vol. 40, no. 2, pp. 544-547, Feb. 2005.

[6] H. J. Lee, D. S. Ha, and S. S. Choi, "A 3 to $5 \mathrm{GHz}$ CMOS UWB LNA with input matching using Miller effect," in Proc. IEEE Solid-State Circuits Conf., Feb. 2006, pp. 731-740.

[7] C. F. Liao and S. I. Liu, "A broadband noise-canceling CMOS LNA for 3.1-10.6-GHz UWB receiver," in Proc. IEEE CICC'05, Sep. 2005, pp. 161-164.

[8] A. Bevilacqu, C. Sandner, A. Gerosa, and A. Neviani, "A fully integrated differential CMOS LNA for 3-5-GHz ultrawideband wireless receivers," IEEE Microw. Wireless Compon. Lett., vol. 16, no. 3, pp. 134-136, Mar. 2006.

[9] C. T. Fu and C. N. Kuo, "3 11-GHz CMOS UWB LNA using dual feedback for broadband matching," in Proc. IEEE Rad. Freq. Integr. Circuits (RFIC) Symp., Jun. 2006, pp. 11-13.

[10] H. W. Chiu, S. S. Lu, and Y. S. Lin, "A $2.17 \mathrm{~dB}$ NF, $5 \mathrm{GHz}$ band monolithic CMOS LNA with $10 \mathrm{~mW}$ dc power consumption," IEEE Trans. Microw. Theory Tech., vol. 53, no. 3, pp. 813-824, Mar. 2005.

[11] D. Barras, F. Ellinger, H. Jäckel, and W. Hirt, "A low supply voltage SiGe LNA for ultra-wideband frontends," IEEE Microw. Wireless Compon. Lett., vol. 14, no. 10, pp. 69-71, Oct. 2004. 